\title{
Integration of a Thin Film PDMS-Based Capacitive Sensor for Tactile Sensing in an Electronic Skin
}

\author{
Sara El-Molla, ${ }^{1,2}$ Andreas Albrecht, ${ }^{1}$ Engin Cagatay, ${ }^{1}$ Philipp Mittendorfer, ${ }^{3}$ \\ Gordon Cheng, ${ }^{3}$ Paolo Lugli, ${ }^{1}$ José F. Salmerón, ${ }^{1}$ and Almudena Rivadeneyra ${ }^{1}$ \\ ${ }^{1}$ Institute for Nanoelectronics, Technical University of Munich, 80333 Munich, Germany \\ ${ }^{2}$ Physics Department, Faculty of Science, Zagazig University, Zagazig 44516, Egypt \\ ${ }^{3}$ Institute for Cognitive Systems, Technical University of Munich, 80333 Munich, Germany \\ Correspondence should be addressed to Almudena Rivadeneyra; almudena.rivadeneyra@tum.de
}

Received 5 January 2016; Accepted 23 February 2016

Academic Editor: Eugenio Martinelli

Copyright (C) 2016 Sara El-Molla et al. This is an open access article distributed under the Creative Commons Attribution License, which permits unrestricted use, distribution, and reproduction in any medium, provided the original work is properly cited.

\begin{abstract}
We present a capacitive force sensor based on a polydimethylsiloxane (PDMS) film integrated into a printed circuit board (PCB) on a flexible substrate whose layout is defined by inkjet printing. The influence of the dielectric thickness on the sensor behavior is presented. The thinner PDMS film of about $45 \mu \mathrm{m}$ shows a sensitivity of up to $3 \mathrm{pF} / \mathrm{N}$ but poorer dynamic response. The dielectrics with thicknesses above $200 \mu \mathrm{m}$ show a significantly reduced sensitivity. The best compromise between sensitivity and dynamic response is found for PDMS film of about $100 \mu \mathrm{m}$, showing about $1.1 \mathrm{pF} / \mathrm{N}$ and less than $15 \mathrm{~s}$ of recovery time. This film is integrated into a flexible PCBS including a microcontroller capable of evaluating the sensor. Interconnects of the circuit are defined by silver nanoparticles deposited by inkjet printing. The working principle of the circuit is demonstrated, proving that this simple approach can be used for artificial skin applications.
\end{abstract}

\section{Introduction}

Over the last decades, there has been an increasing interest in the development of tactile sensing electronic devices able to provide "sense of touch" $[1,2]$. In humans, the skin is the physical frontier between our environment and ourselves and helps us to interact in a successful way with the outer world. Apart from being a protective layer, our skin helps us to collect information of our surrounding environment and to perform essential tasks, such as motion control or grasping objects [3]. To do that, human skin is composed of a large number of different detectors embedded in our skin at different levels and with variable densities. Those detectors are in charge of detecting different modalities: force, pressure, strain, light, and temperature. The collected information is converted into electrical signals that are processed by the central or peripheral nervous system in a more complex way. In fact, the human skin acts as an interface with the outside environment [4].
Printed Electronics (PE) makes use of traditional printing techniques, that is, screen-printing, gravure, inkjet printing, and so forth, to fabricate electronic circuitry. The recent advances in materials and fabrication techniques in $\mathrm{PE}$ are leading to a complete new generation of electronic devices with remarkable new features in traditional electronics, like flexibility, lightness, ultra-cost-effectiveness, and biodegradability. These properties are mandatory for artificial skin due to the mechanical requirements, weight restrictions, and the need for the device to be conformal with the shape of the object to be attached. Moreover, printing has the capability of manufacturing large products, up to several tens of meters wide. Sensor modules capable of performing tactile sensing have been already developed. Tactile skin devices should include measurements of strain, shear forces, pressure, and vibration [5-8].

In this framework, capacitive sensors are especially interesting and they have been employed to measure normal forces, shear forces, and strain $[6,9,10]$. They can 
be fabricated with small dimensions, providing a high spatial resolution. A combination of multiple sensors can be employed to detect forces of different types in all directions [11]. Several groups have developed tactile sensor matrices or arrays $[12,13]$ and even some research groups made the attempt to completely cover a humanoid with artificial skin $[14,15]$. In literature, several examples of the integration of an electronic skin for humanoids can be found [4, 16, 17].

In this work, we present a study of a simple but reliable capacitive sensor based on the elastomer polydimethylsiloxane (PDMS) film and its integration into a flexible printed circuit board (PCB) manufactured by inkjet printing. The mechanical properties of PDMS films have been already studied [18-21]. Thanks to its mechanical characteristics and its relative ease of manufacturing, several authors have used PDMS as the core material of force sensors. Mannsfeld et al. developed flexible capacitive pressure sensors based on microstructured thin PDMS films. They are capable of tuning the pressure sensitivity by using different microstructured patterns, such as lines and pyramids [22]. Lipomi et al. introduced skin-like capacitive pressure and strain sensors where the silicone elastomer Ecoflex is placed between transparent elastic films of carbon nanotube (CNT) and PDMS, acting as electrodes [9]. Lei et al. have shown a flexible capacitive pressure sensor based on PDMS developed for plantar pressure measurement in biomechanical applications [23]. The designed sensor is capable of detecting up to $945 \mathrm{kPa}$ and consists of four layers: lower layer, dielectric layer, upper layer, and bump layer. A work from Woo et al. presented a capacitive force sensor array based on a micro-patterned film of a conductive CNT-doped PDMS produced by a combination of soft-lithographic replication and contact printing [24]. Flexible capacitive tactile sensors with microstructured PDMS as dielectric material and utilizing spray deposited CNT thin films as transparent conductors on polyimide substrates have been reported [25]. The sensor can detect forces below $10 \mathrm{mN}$ and a variation in capacitance of up to $20 \%$ for $1 \mathrm{~N}$ of applied force can be achieved with good long-term reliability. There are further studies in literature where a mixture of PDMS and CNT is used as resistive force sensor [26-28] but the capacitive approach is desirable for PCB design due to its high speed response and long-lasting reliability [29].

In the approach presented here, a capacitive sensor is characterized and integrated together with a microcontroller and other devices on a flexible substrate where interconnects are defined by inkjet printing of silver nanoparticles. The force sensor is a parallel plate capacitor whose dielectric is a PDMS film. The performances of different PDMS thicknesses have been compared with the aim of finding the most suitable one. This work demonstrates the feasibility of an unstructured PDMS film as a capacitive sensor integrated on a flexible PCB, bringing closer the development of artificial skin solutions.

\section{Materials and Methods}

2.1. Materials. The flexible substrate was Kapton with copper laminated onto the top surface (Pyralux ${ }^{\circledR}$ from DuPont, USA) that was used as one of the electrodes of our device.
The other electrode was made of electrically conductive ink (DGP 40LT-15C from ANP Co., USA) with about 30-35\% of silver nanoparticles and triethylene glycol monoethyl ether (TMGE) as solvent. The dielectric spacer used was the polydimethylsiloxane (PDMS) which consists of two components base and curing agent (Sylgard 184, Dow Corning).

2.2. Fabrication Process. The PDMS films were prepared by mixing the base and the curing agent in a weight ratio of $10: 1$, respectively. A mechanical stirring for the mixture was applied for $10 \mathrm{~min}$. Then, the mixture is desiccated for $30 \mathrm{~min}$ to remove entrapped air bubbles resulting from stirring. The viscous PDMS mixture was then directly deposited onto the copper face of Kapton and spin-coated to reach the desired thickness of PDMS and get a uniform thin film at the same time. Spin coating at $10 \mathrm{rps}$ for $30 \mathrm{~s}$ and $25 \mathrm{rps}$ for $60 \mathrm{~s}$ resulted in a layer thickness of about $100 \mu \mathrm{m}$ and $45 \mu \mathrm{m}$, respectively. Thicker films were composed of multiple spin-coated layers. Then, the film was desiccated again for $10 \mathrm{~min}$ to remove any enclosed air bubbles. Afterwards the PDMS is cured by placing the sample on a hotplate at $100^{\circ} \mathrm{C}$ for $60 \mathrm{~min}$. The second electrode was inkjet-printed on the top layer of PDMS with a DMP-2831 ${ }^{\mathrm{TM}}$ Dimatix printer (Fujifilm Dimatix Inc., Santa Clara, USA). The substrate temperature was fixed at $60^{\circ} \mathrm{C}$ during printing. The top electrode consisted of two consecutive printed layers. A drop space of $40 \mu \mathrm{m}$ was settled in the printer for $80 \mu \mathrm{m}$ landed diameter drops followed by a drying step at $80^{\circ} \mathrm{C}$ for $10 \mathrm{~min}$.

After the drying of the silver layer at $80^{\circ} \mathrm{C}$ for $10 \mathrm{~min}$, a photonic sintering (Sinteron 2010 from Xenon, USA) was conducted with a voltage of $2.5 \mathrm{kV}$ and 15 pulses of $500 \mu$ s to ensure a good resistivity of the printed patterns. Finally, two wires were glued to the electrodes using silver-filled epoxy EPO-TEK ${ }^{\circledR} \mathrm{H} 20 \mathrm{E}$ (Epoxy Technology, Inc., Billerica, USA) and the device was dried at $60^{\circ} \mathrm{C}$ for $60 \mathrm{~min}$.

2.3. Characterization. The thicknesses of the PDMS films and silver electrodes were measured with a Dektak XT ${ }^{\mathrm{TM}}$ Stimulus Surface Profiling System (Bruker Corporation, Coventry, UK). Sheet resistance measurements were conducted on the printed electrode with a self-made linear four-point probe in combination with a Keithley ACS 2600 (Germering, Germany). A correction factor of 0.651 was calculated and applied to remove the effect of limited boundaries according to Smits [30].

The characterization of the force was performed as in [25]. A voice coil actuator VM2836-180 from Geeplus (Beckenham, UK) was used to generate the normal force $F_{z}$. By applying a current to the coil inside the actuator, a magnetic field was induced and the movable coil was displaced with respect to the permanent magnet. The coil of the actuator was attached to a metallic bar of $5.10 \pm 0.05 \mathrm{~mm}$ diameter, which transfers the movement mechanically to the tactile sensor. A piezoresistive PK 88870 force sensor (FSG Series from Honeywell, Offenbach, Germany) was used to obtain feedback control on $F_{z}$. The driving current was supplied to the actuator through a Keithley 2602 SourceMeter (Germering, Germany). The capacitance and series resistance of the fabricated sensors were continuously monitored using an 


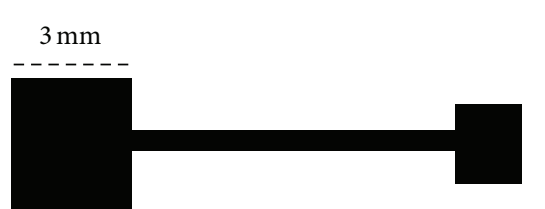

(a)

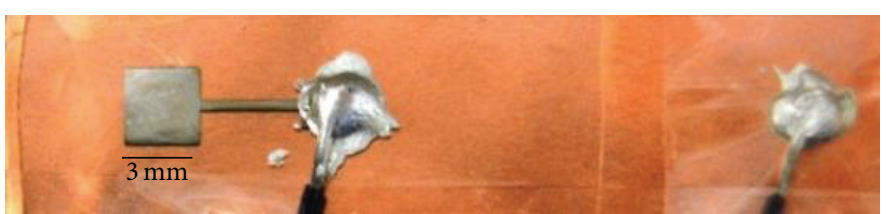

(b)



(d)

FIGURE 1: (a) Layout of the printed top electrode; (b) image of the fabricated sensor; (c) cross-sectional view of the sensor; (d) microscopic image of silver NPs over PDMS.

Agilent E4980A Precision LCR Meter (Böblingen, Germany) at $100 \mathrm{kHz}$. The entire setup was controlled and data were recorded using the software LabVIEW (National Instruments Corporation, Texas, USA).

\section{Results and Discussion}

3.1. Physical Characterization. The structure with the shape presented in Figure 1(a) was printed on top of the dry PDMS, resulting in the sensor shown in Figure 1(b). The printed structures were designed with contacts of $(3 \mathrm{~mm} \times 3 \mathrm{~mm})$ and $(1 \mathrm{~mm} \times 1 \mathrm{~mm})$, where the distance between contacts was $1 \mathrm{~cm}$ with $1 \mathrm{~mm}$ width. Figure 1(c) illustrates the layers that composed this sensor.

After performing the photonic sintering process, the sheet resistance of the printed electrode was measured to be about $19.2 \pm 2.2 \Omega / \square$. An optical microscope image of the printed layer on top of the PDMS is depicted in Figure 1(d). The thickness of the printed silver on the PDMS film is shown in Figure 2. The typical coffee ring effect of printed layers can also be seen in the same figure. Surface profilometer measurements revealed the thickness of the printed silver layer on PDMS to be about $4290 \pm 200 \mathrm{~nm}$. This is about one order of magnitude higher than silver layers on other substrates printed with the same drop spacing and drop waveform [31, 32]. This huge difference in height cannot be explained with a reduced packing density of the silver nanoparticles. The only other material in direct contact with the PDMS is the solvent of the ink. The used ink possesses around 95\% (vol) of TGME that leads to a wet ink layer of approximately 6-7 $\mu \mathrm{m}$ after printing [33], which is close to the thicknesses we measured. We can exclude the possibility of entrapped solvent in the layer after drying by visual inspection of the silver-colored layer (Figures 1(b) and 1(c)).
Thus, we could assume that the increased height of the printed silver tracks on PDMS is related to a swelling of the PDMS after solvent absorption. Among others, Lee et al. investigated the effect of many solvents on swelling using an immersed piece of PDMS [34] and found a swelling behavior for almost all tested solvents with a Hansen total solubility parameter close to PDMS. According to Dow Chemical [35] and the calculation method of Lee et al. this parameter is 10.1 for TGME. Other solvents having this parameter have a swelling ratio between $103 \%$ and $121 \%$. The higher ratio leads to the swelling of the PDMS of about $4 \mu \mathrm{m}$ already if the top $20 \mu \mathrm{m}$ of PDMS is saturated with solvent. The swelling of the PDMS also explains the small increase in height next to the printed layers in Figure 2 between 0.5 and $0.6 \mathrm{~mm}$.

3.2. Capacitive Sensors. Different PDMS films were prepared with different thickness values. Figure 3 illustrates the sensitivity $(S)$ of the device defined as the change in capacitance $\Delta C$ in $\mathrm{pF}$ divided by the change in force $\Delta F$ in $\mathrm{N}$ :

$$
S(\mathrm{pF} / \mathrm{N})=\frac{\Delta C}{\Delta F}
$$

In all cases, we measured $\Delta C$ at $1 \mathrm{~N}$ force $(\Delta F)$ and looked at $\Delta C$ after applying the force for $1 \mathrm{~min}$.

As can be seen, the sensor sensitivity decreases exponentially with the increase in the PDMS thickness. This result is in agreement with previous reports in literature $[18,22]$. Our objective here is to achieve a simple force sensor that can be easily integrated into a flexible PCB. Therefore, our sensor must be sensitive enough to be measured by a standard microcontroller and fast enough to do tracking of the applied force. At first glance, a thinner dielectric film leads to a higher sensitivity and, thus, a better performance. 


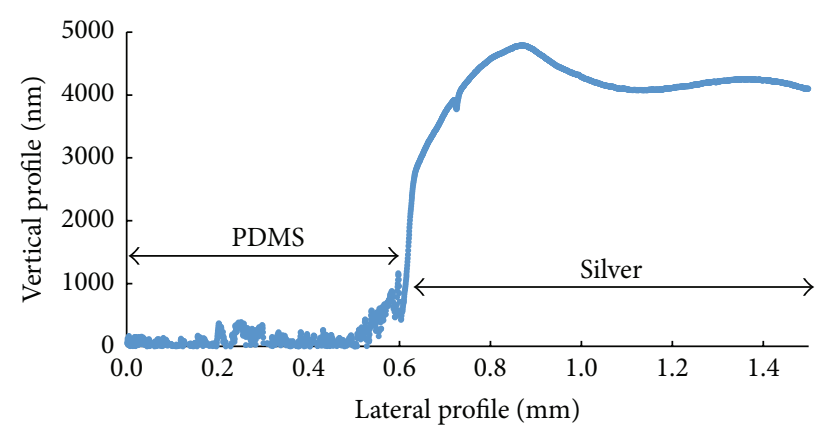

Figure 2: Profile of the printed silver layer on top of the PDMS.

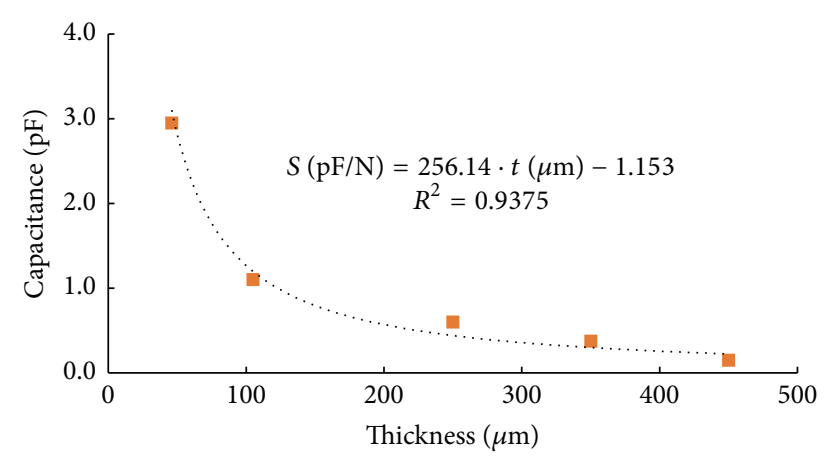

FIgURE 3: Sensitivity as a function of PDMS thickness.

However, when we analyzed the dynamic response of the thinnest film $(\sim 45 \mu \mathrm{m})$, the time required to recover its initial capacitance value was much higher than the time required by the film of about $100 \mu \mathrm{m}$ thickness. The thinnest layer requires more time to recover, while the $100 \mu \mathrm{m}$ film needs less than $15 \mathrm{~s}$. Furthermore, the $100 \mu \mathrm{m}$ thick PDMS film used in this work has lower hysteresis in comparison to the one with a lower thickness. The reason behind is the fact that unstructured films, which are thinner than several hundred micrometers, are subject to significant viscoelastic creep. On the other hand, thicker unstructured PDMS films are nearly fully elastic under pressures lower than $100 \mathrm{kPa}$. As a result, thin PDMS films have higher relaxation times compared to thick films, which accounts for a higher hysteresis during device operation [22]. For this reason, we selected the device with a PDMS film thickness of $100 \mu \mathrm{m}$, which presents the best compromise between nominal capacitance $(\sim 5 \mathrm{pF})$ and change under applied force ( $>20 \%)$.

Figure 4 illustrates the response of the selected sensor $(\sim 100 \mu \mathrm{m})$ when applying an increasing force of $50 \mathrm{mN}$ every $30 \mathrm{~s}$. This behavior can be approximated by a rational function with one zero and one pole; see (2). In particular, the parameters are $a=5.81 \mathrm{pF}, b=331.70$, and $c=66.55$ with $R^{2}=0.9843$. The response can also be approximated by linearization when we define the suitable force ranges. For instance, in $30-150 \mathrm{mN}$ force range the sensitivity is about $3.7 \mathrm{fF} / \mathrm{mN}$, while this sensitivity decreases to $0.7 \mathrm{fF} / \mathrm{mN}$ in $150-450 \mathrm{mN}$ range and to $0.06 \mathrm{fF} / \mathrm{mN}$ at higher forces

$$
C(\mathrm{pF})=\frac{a \cdot F(\mathrm{mN})+b}{F(\mathrm{mN})+c} .
$$



FIGURE 4: Capacitance versus applied force for about $100 \mu \mathrm{m}$ PDMS thickness.

In order to observe the dynamic response of this sensor and its hysteresis and reproducibility, we performed different tests presented in Figure 5. Figure 5(a) illustrates the high reproducibility of the sensor as well as its fast time response to the force applied, although the recovery time is slightly longer than the response time.

As can be seen in Figure 5(b), the sensor goes back to its initial capacitance value if there is no force applied with an error less than $3 \%$ at the different force steps applied. If we compare this response with the one reported by Cagatay et al. [25] for a PDMS film of about $110 \mu \mathrm{m}$ and pillar of about $30 \mu \mathrm{m}$, we observe similar capacitance values under no force. The sensitivity is about $10 \%$ lower in the sensor described here, but the fabrication procedure and the integration on a final system are much easier.

3.3. Sensor Integration. After showing the performance of the standalone sensor, we integrated it into a hybrid system of an inkjet-printed circuit board assembled with conventional surface mount components (Figure 6). The circuitry was printed with silver nanoparticle inkjet ink on a coated polyethylene terephthalate (PET) based substrate (Novele from Novacentrix, USA). From top left to bottom right, it contains a connector for programming the microcontroller $(\mu \mathrm{C})$ and retrieving the measured values, four light emitting diodes (LEDs) with their corresponding serial resistor (R), and the sensor electrode. Above the sensor electrode, a piece of a $100 \mu \mathrm{m}$ thick PDMS film was placed. Although we have demonstrated the possibility of direct printing the electrode on the PDMS, after pressing several times the silver layer on PDMS without any protection, this layer starts to show cracks. In order to avoid this issue and ensure longer durability of the system, we decided to employ another PET substrate with an inkjet-printed electrode on top of the PDMS film with the electrode facing down. These two electrodes touching the PDMS layer on each side form the capacitive sensor. All electrical connections were done with electrically conductive epoxy. 


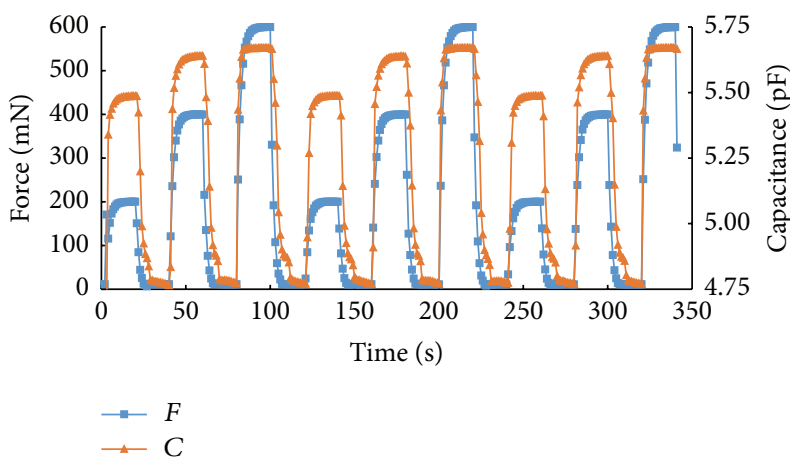

(a)

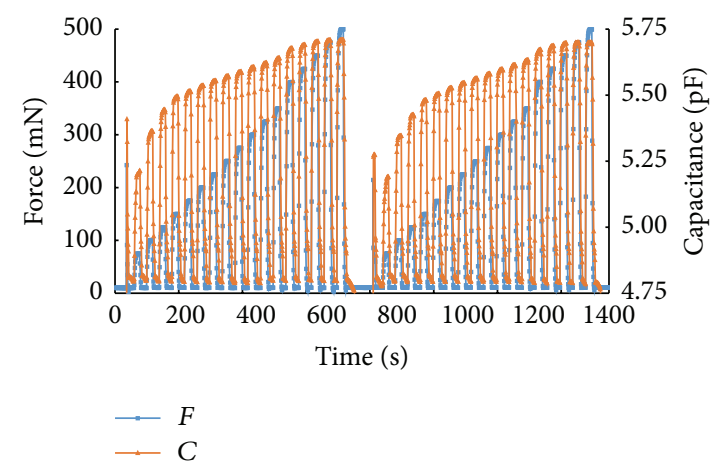

(b)

FIGURE 5: Dynamic response of the selected sensor (about $100 \mu \mathrm{m}$ PDMS thickness).

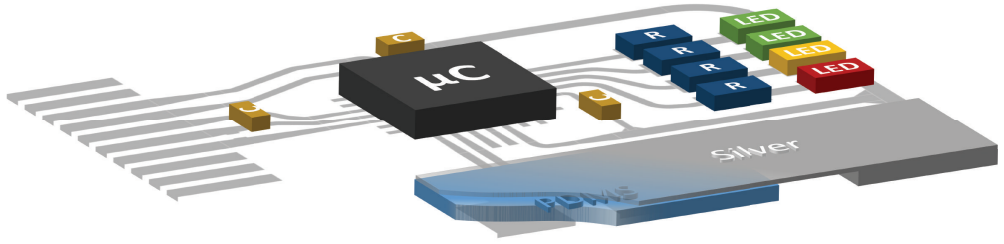

(a)

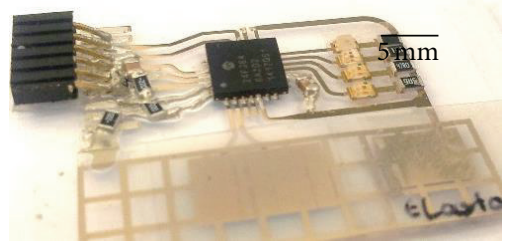

(b)

FIGURE 6: (a) Schematic and photograph of hybrid integrated system with inkjet-printed silver layers; (b) surface mount devices (SMD) and PDMS film.

The microcontroller PIC24FJ64GA202 (Microchip) is equipped with a charge time measurement unit (CMTU), which was used to measure the capacitive sensor. We used a current $(I)$ of $55 \mu \mathrm{A}$ to charge the capacitor for $1.5 \mu \mathrm{s}(t)$ with the charge $Q$. The resulting voltage $V$ at the electrodes was then converted to a digital value $D$ using the internal 12 bit analog-to-digital converter of the microcontroller with the reference voltage $\left(V_{\text {ref }}\right)$ at $3.25 \mathrm{~V}$. This procedure allows us to calculate the measured capacitance following the equation

$$
C=\frac{Q}{V}=\frac{I \cdot t}{D \cdot V_{\text {ref }} / 2^{12}}
$$

The measured force profile consists of a ramp with increasing force. Every $30 \mathrm{~s}$ the force was increased by $20 \%$ from $5 \mathrm{mN}$ to $1000 \mathrm{mN}$. Force and average capacitance values were taken $25 \mathrm{~s}$ after each step to allow both values to stabilize. We measured the voltage 100 times and averaged it to increase the signal-to-noise ratio. Figure 7 shows a very small change of capacitance and a clear trend to higher capacitance at higher force. The relation between the capacitance $C$ and the force $F$ can be approximated by (2), where the parameter values are $a=28.83 \mathrm{pF}, b=126.1$, and $c=4.414$ at $R^{2}=0.9938$.

In comparison to direct capacitive measurements of the sensor, the microcontroller includes the parasitic capacitances of its input pins and the wires to the sensor. Based on the presented results above, this parasitic capacitance is in the order of $24 \mathrm{pF}$.

The relative change in the capacitance in this experiment is very small and touches the limits of the measurement capabilities of the microcontroller. One reason is the small

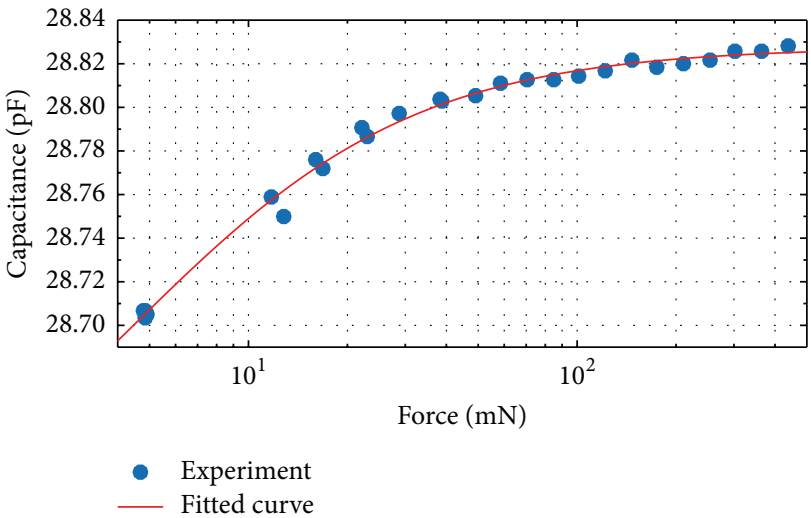

FIGURE 7: Capacitance between sensor electrodes versus applied force and fitted curve.

sensor capacitance compared to the parasitic capacitance. This drawback could be overcome by using a strategy similar to the one presented by Toth and Meijer [36]. As another possibility of improving the system performance, it is to enhance the sensor capacitance to raise sensitivity. It is suggested to increase the electrode area to the maximum size suitable for the application and/or use microstructured PDMS films that present higher sensitivities. The relative change in the sensor capacitance may be improved by using microstructured PDMS films that achieve a higher sensitivity by changing the average dielectric constant between the sensor electrodes. 


\section{Conclusions}

In this work, a capacitive force sensor based on polydimethylsiloxane (PDMS) is presented. We have prepared different PDMS films by varying the film thickness. These films have been placed on a polyimide foil with copper layer, acting as bottom electrode, and a silver electrode has been printed on the other side of the PDMS, acting as top electrode. It has been observed that the thickness of the printed silver is 10 times higher than the one achieved in other substrates for the same ink with the same drop spacing and drop waveform. This increased height of the printed silver tracks on PDMS can be attributed to swelling of the PDMS after solvent absorption.

The PDMS film with about $45 \mu \mathrm{m}$ thickness presents a sensitivity of about $3 \mathrm{pF} / \mathrm{N}$ or a change in capacitance of about $45 \%$, whereas when the thickness increases the sensitivity is drastically reduced. Although thinner films result in higher sensitivities, the dynamic response is penalized and more time is required to recover the initial value. We found that the best compromise between the sensor sensitivity and its dynamic response is for thicknesses about $100 \mu \mathrm{m}$. For this kind of PDMS films, a nonlinear sensitivity of about $1.1 \mathrm{pF} / \mathrm{N}$ is achieved with a response time less than $15 \mathrm{~s}$ and no substantial hysteresis in its response.

The best candidate $(\sim 100 \mu \mathrm{m})$ has been characterized and integrated together with a microcontroller and other components on a flexible substrate, where interconnects are defined by inkjet printing of silver nanoparticles, proving the suitability of this technology to integrate those sensors in more complex printed circuits for artificial skin applications.

\section{Competing Interests}

The authors declare that they have no competing interests.

\section{Acknowledgments}

This work was partially supported by the European Union through FP7-PEOPLE-2012-ITN 316832-OLIMPIA, FP7PEOPLE-2013 607896-OrgBIO, and PITN-GA-2012-317488CONTEST. This work was also partially funded by the TUM Graduate School.

\section{References}

[1] M. L. Hammock, A. Chortos, B. C.-K. Tee, J. B.-H. Tok, and Z. Bao, "25th anniversary article: the evolution of electronic skin (e-skin): a brief history, design considerations, and recent progress," Advanced Materials, vol. 25, no. 42, pp. 5997-6038, 2013.

[2] A. Chortos and Z. Bao, "Skin-inspired electronic devices," Materials Today, vol. 17, no. 7, pp. 321-331, 2014.

[3] R. S. Dahiya, P. Mittendorfer, M. Valle, G. Cheng, and V. J. Lumelsky, "Directions toward effective utilization of tactile skin: a review, IEEE Sensors Journal, vol. 13, no. 11, pp. 4121-4138, 2013.

[4] R. S. Dahiya, G. Metta, M. Valle, and G. Sandini, "Tactile sensing-from humans to humanoids," IEEE Transactions on Robotics, vol. 26, no. 1, pp. 1-20, 2010.
[5] M. Hussain, Y.-H. Choa, and K. Niihara, "Conductive rubber materials for pressure sensors," Journal of Materials Science Letters, vol. 20, no. 6, pp. 525-527, 2001.

[6] K. Kanao, S. Harada, Y. Yamamoto et al., "Highly selective flexible tactile strain and temperature sensors against substrate bending for an artificial skin," RSC Advances, vol. 5, no. 38, pp. 30170-30174, 2015.

[7] P. Mittendorfer and G. Cheng, "Humanoid multimodal tactilesensing modules," IEEE Transactions on Robotics, vol. 27, no. 3, pp. 401-410, 2011.

[8] E. Cagatay, P. Mittendorfer, A. Abdellah, G. Cheng, and P. Lugli, "Integrating CNT force sensors into a multi-modal modular electronic skin," in Proceedings of the IEEE 15th International Conference on Nanotechnology (IEEE-NANO '15), pp. 12991302, IEEE, Rome, Italy, July 2015.

[9] D. J. Lipomi, M. Vosgueritchian, B. C.-K. Tee et al., "Skin-like pressure and strain sensors based on transparent elastic films of carbon nanotubes," Nature Nanotechnology, vol. 6, no. 12, pp. 788-792, 2011.

[10] B. B. Narakathu, A. Eshkeiti, A. S. G. Reddy et al., "A novel fully printed and flexible capacitive pressure sensor," in Proceedings of the IEEE Sensors Applications Symposium (SAS '12), pp. 1-4, Taipei, Taiwan, October 2012.

[11] S. Harada, K. Kanao, Y. Yamamoto, T. Arie, S. Akita, and K. Takei, "Fully printed flexible fingerprint-like three-axis tactile and slip force and temperature sensors for artificial skin," ACS Nano, vol. 8, no. 12, pp. 12851-12857, 2014.

[12] G. Schwartz, B. C.-K. Tee, J. Mei et al., "Flexible polymer transistors with high pressure sensitivity for application in electronic skin and health monitoring," Nature Communications, vol. 4, article 1859, 2013.

[13] M. Ying, A. P. Bonifas, N. S. Lu et al., "Silicon nanomembranes for fingertip electronics," Nanotechnology, vol. 23, p. 7, 2012.

[14] Y. Fujimori, Y. Ohmura, T. Harada, and Y. Kuniyoshi, "Wearable motion capture suit with full-body tactile sensors," in Proceedings of the IEEE International Conference on Robotics and Automation (ICRA '09), pp. 3186-3193, Kobe, Japan, May 2009.

[15] M. Hayashi, T. Sagisaka, Y. Ishizaka, T. Yoshikai, and M. Inaba, "Development of functional whole-body flesh with distributed three-axis force sensors to enable close interaction by humanoids," in Proceedings of the IEEE/RSJ International Conference on Intelligent Robots and Systems (IROS '07), pp. 3610-3615, IEEE, San Diego, Calif, USA, November 2007.

[16] J. Engel, J. Chen, Z. Fan, and C. Liu, "Polymer micromachined multimodal tactile sensors," Sensors and Actuators A: Physical, vol. 117, no. 1, pp. 50-61, 2005.

[17] T. Someya, T. Sekitani, S. Iba, Y. Kato, H. Kawaguchi, and T. Sakurai, "A large-area, flexible pressure sensor matrix with organic field-effect transistors for artificial skin applications," Proceedings of the National Academy of Sciences of the United States of America, vol. 101, no. 27, pp. 9966-9970, 2004.

[18] J. C. Lötters, W. Olthuis, P. H. Veltink, and P. Bergveld, "The mechanical properties of the rubber elastic polymer polydimethylsiloxane for sensor applications," Journal of Micromechanics and Microengineering, vol. 7, no. 3, pp. 145-147, 1997.

[19] I. D. Johnston, D. K. McCluskey, C. K. L. Tan, and M. C. Tracey, "Mechanical characterization of bulk Sylgard 184 for microfluidics and microengineering," Journal of Micromechanics and Microengineering, vol. 24, no. 3, Article ID 035017, 2014.

[20] A. Mata, A. J. Fleischman, and S. Roy, "Characterization of polydimethylsiloxane (PDMS) properties for biomedical 
micro/nanosystems," Biomedical Microdevices, vol. 7, no. 4, pp. 281-293, 2005.

[21] M. Liu, J. Sun, Y. Sun, C. Bock, and Q. Chen, "Thicknessdependent mechanical properties of polydimethylsiloxane membranes," Journal of Micromechanics and Microengineering, vol. 19, no. 3, Article ID 035028, 2009.

[22] S. C. B. Mannsfeld, B. C.-K. Tee, R. M. Stoltenberg et al., "Highly sensitive flexible pressure sensors with microstructured rubber dielectric layers," Nature Materials, vol. 9, no. 10, pp. 859-864, 2010.

[23] K. F. Lei, K.-F. Lee, and M.-Y. Lee, "Development of a flexible PDMS capacitive pressure sensor for plantar pressure measurement," Microelectronic Engineering, vol. 99, pp. 1-5, 2012.

[24] S.-J. Woo, J.-H. Kong, D.-G. Kim, and J.-M. Kim, "A thin allelastomeric capacitive pressure sensor array based on microcontact printed elastic conductors," The Journal of Materials Chemistry C, vol. 2, no. 22, pp. 4415-4422, 2014.

[25] E. Cagatay, P. Kohler, P. Lugli, and A. Abdellah, "Flexible capacitive tactile sensors based on carbon nanotube thin films," IEEE Sensors Journal, vol. 15, no. 6, pp. 3225-3233, 2015.

[26] J.-E. Han, D. Kim, and K.-S. Yun, "All-polymer hair structure with embedded three-dimensional piezoresistive force sensors," Sensors and Actuators A: Physical, vol. 188, pp. 89-94, 2012.

[27] S. Khan, L. Lorenzelli, and R. S. Dahiya, "Bendable piezoresistive sensors by screen printing MWCNT/PDMS composites on flexible substrates," in Proceedings of the 10th Conference on Ph.D. Research in Microelectronics and Electronics (PRIME '14), pp. 1-4, IEEE, Grenoble, France, June 2014.

[28] Y. Jung, D.-G. Lee, J. Park, H. Ko, and H. Lim, "Piezoresistive tactile sensor discriminating multidirectional forces," Sensors, vol. 15, no. 10, pp. 25463-25473, 2015.

[29] L. K. Baxter, Capacitive Sensors: Design and Applications, John Wiley \& Sons, New York, NY, USA, 1996.

[30] F. M. Smits, "Measurement of sheet resistivities with the fourpoint probe," Bell System Technical Journal, vol. 37, no. 3, pp. 711$718,1958$.

[31] A. Rivadeneyra, J. Fernández-Salmerón, M. Agudo, J. A. LópezVillanueva, L. F. Capitan-Vallvey, and A. J. Palma, "Design and characterization of a low thermal drift capacitive humidity sensor by inkjet-printing," Sensors and Actuators B: Chemical, vol. 195, pp. 123-131, 2014.

[32] A. Rivadeneyra, J. Fernández-Salmerón, M. Agudo-Acemel, J. A. López-Villanueva, A. J. Palma, and L. F. Capitan-Vallvey, "A printed capacitive-resistive double sensor for toluene and moisture sensing," Sensors and Actuators B: Chemical, vol. 210, pp. 542-549, 2015.

[33] J. F. Salmerón, F. Molina-Lopez, D. Briand et al., "Properties and printability of inkjet and screen-printed silver patterns for RFID antennas," Journal of Electronic Materials, vol. 43, no. 2, pp. 604617, 2014.

[34] J. N. Lee, C. Park, and G. M. Whitesides, "Solvent compatibility of poly(dimethylsiloxane)-based microfluidic devices," Analytical Chemistry, vol. 75, no. 23, pp. 6544-6554, 2003.

[35] DOW P-Series Glycol Ethers, December 2015, http://msdssearch.dow.com/PublishedLiteratureDOWCOM/dh_012d/0901b8038012d976.pdf.

[36] F. N. Toth and G. C. M. Meijer, "A low-cost, smart capacitive position sensor," IEEE Transactions on Instrumentation and Measurement, vol. 41, no. 6, pp. 1041-1044, 1992. 




\section{Enfincering}
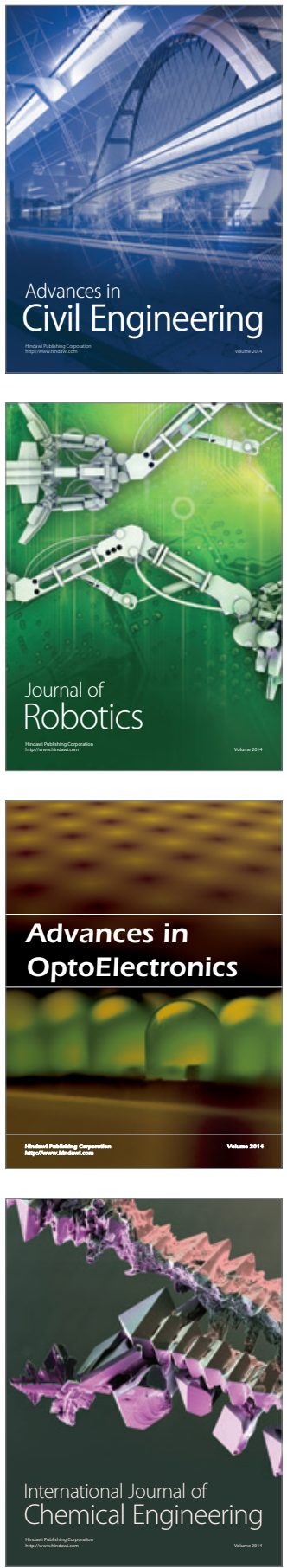

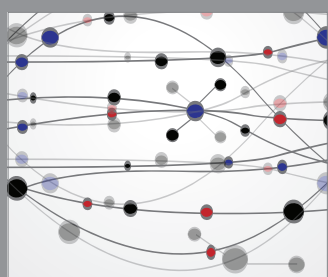

The Scientific World Journal

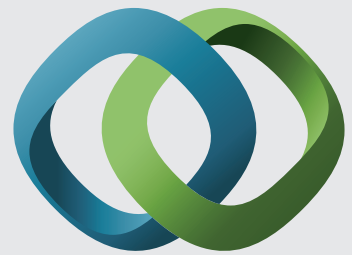

\section{Hindawi}

Submit your manuscripts at

http://www.hindawi.com
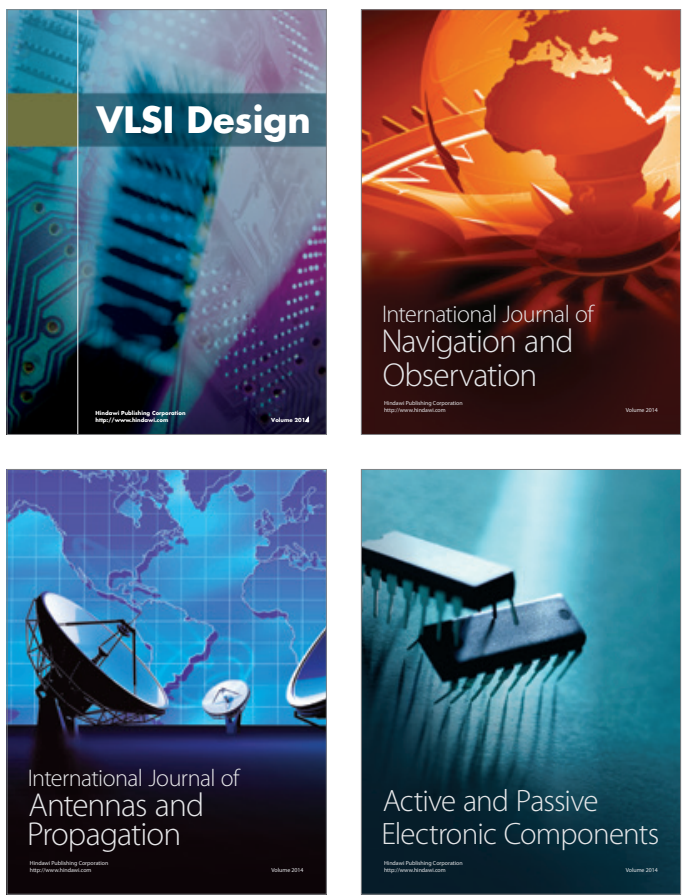
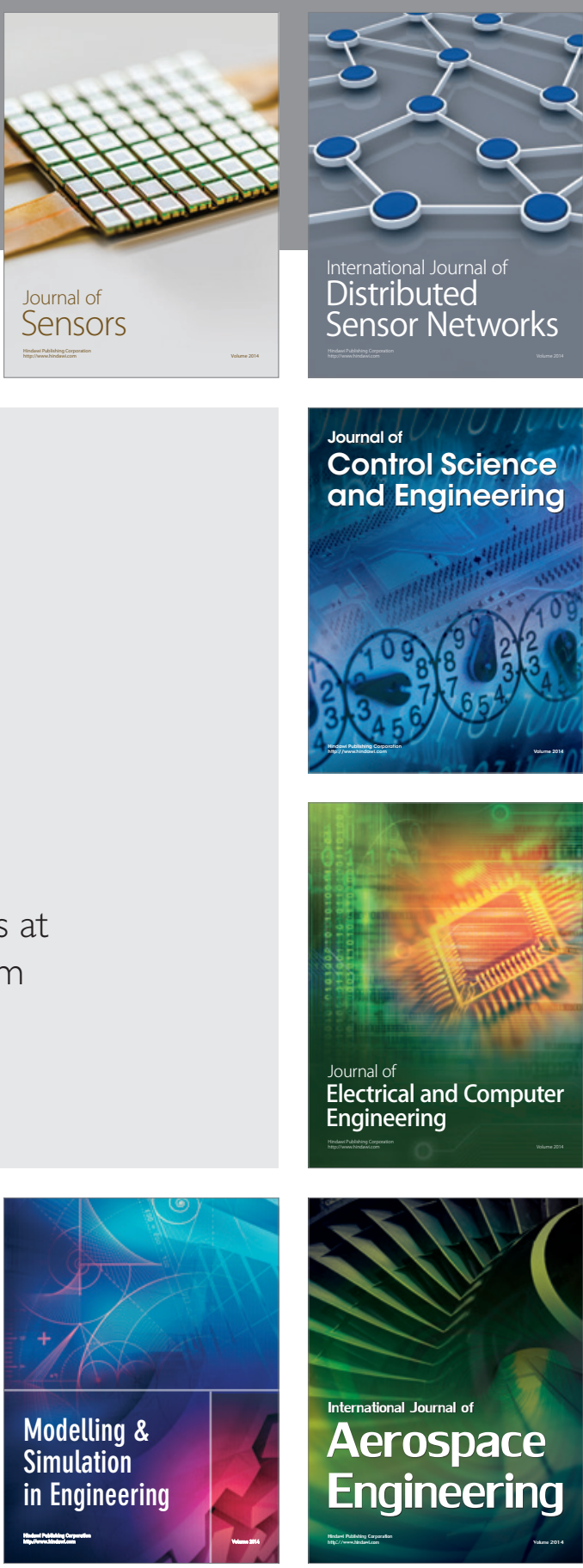

International Journal of

Distributed

Sensor Networks

Journal of

Control Science

and Engineering
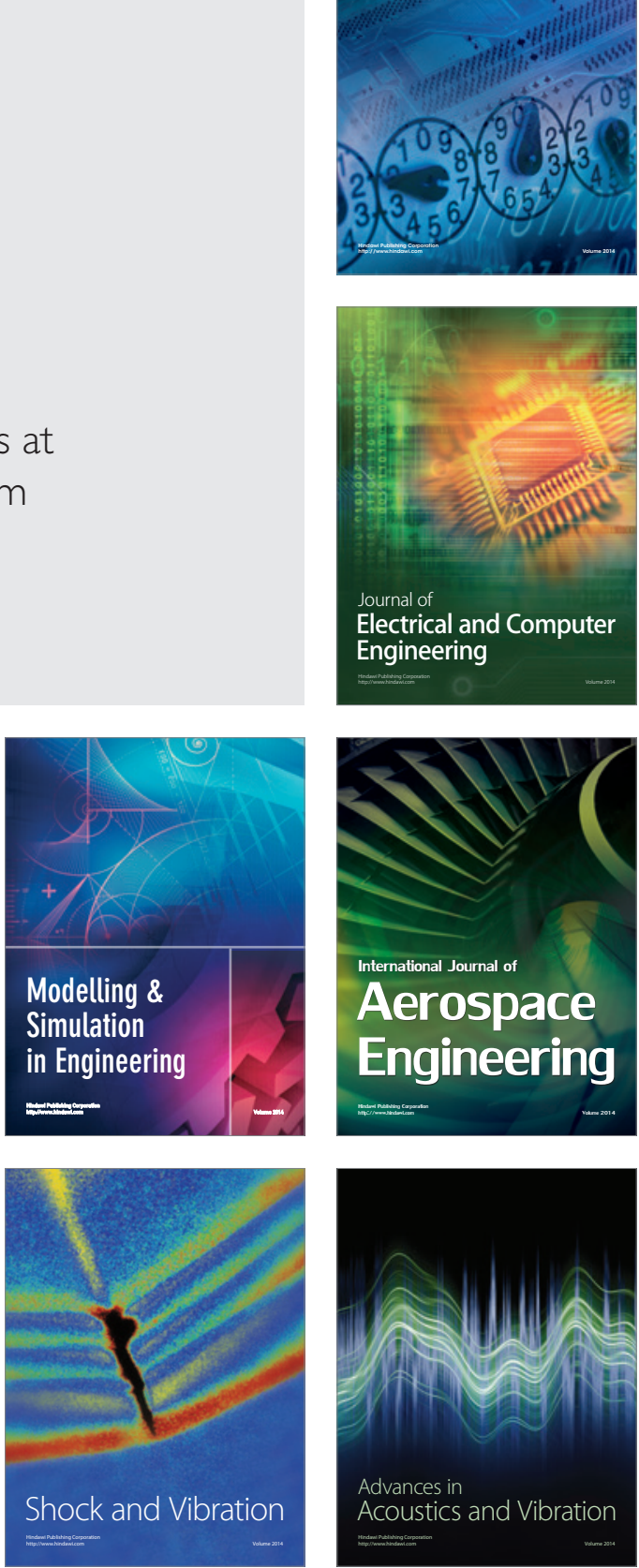